\title{
Astronomy in the Former Soviet Union
}

\begin{abstract}
At the invitation of the SOC, Drs. Y. Balega and A. Tokovinin gave informal presentations concerning the current situation of astronomers in the former USSR. Their experiences provided "snapshots" of particular aspects of Russian science at this critical time in history, and we consider their words and the ensuing discussion to be an important aspect of this Colloquium - Editors
\end{abstract}

BALEGA: The scientific institutes located outside major area of population are being hit hardest by the serious economic crisis in the former Soviet Union. Among these are the Special Astrophysical Observatory of the Academy of Sciences. The name "Special" reflects the fact that the observatory has two large instruments at its disposal: the RATAN-600 Radio Telescope of the Academy of Sciences and the optical 6-m reflector BTA or Big Azimuthal Telescope. The observatory is located near the Zelenchuk River in the mountainous area of the Karachai-Cherkesia Republic, which is a part of the Russian Federation. After the collapse of the USSR, the observatory became the property of Russia.

The main source of funding of the observatory has been a basic allowance from the Academy of Sciences which was about 8-10 million rubles (MR) in the beginning of "perestroika". From this money, we could support a staff of approximately 600 people ( 120 of whom are researchers), maintain the two telescopes and provide astrophysical research programs. The existence of the village, where the staff lives, was also supported through this funding. During 1992, the situation in the observatory became critical. We have very limited communication with the outside world now. The cost of maintenance of the observatory due to "astronomical" prices (increased 30-100 times) has become too high to be covered by the Academy. According to the estimates made by our administration, in 1992 the fuel and electricity alone will cost a minimum of $60-80 \mathrm{MR}$. In the same time we expect an annual budget of about $40 \mathrm{MR}$. We have stopped all innovations and big research programs.

At present we are searching for a simple means of survival. One of the principal proposals is to organize an independent international observatory that would be available for any country, using the existing SAO structure. Proposals have already been discussed in the Russian Ministry of Science. We believe that the telescopes should be considered the property of world science, not the sole property of Russia. SAO is one of the world's largest observatories, and has the highest latitude among them. The new observatory could be a complementary observatory to ESO, according to our proposal. One element of the national VLBI network is under construction in Zelenchuk. It will have a broad-band output to international communication systems and can be used in future for international VLBI experiments. Only a few percent of the ESO annual budget could stop the degradation process of SAO and give the astronomical community unique instruments for big science. There are already many joint projects in our observatory with European and American research groups: measurements of the cosmological radio background, a search for the most distant radio galaxies, solar investigations, optical speckle interferometry, multi-object deep field 
spectroscopy, etc. All the projects could be integrated into one structure - the International Northern Observatory. Our contribution is the cost of the observatory (about $500 \mathrm{MR}$ ) and $50 \%$ of the observing time at the two telescopes.

We have several strong groups ready for new initiatives in optical and radio astrononiy. New technologies and new image formation approaches can be incorporated easily. The financial support of the activity under this new cooperative observatory would be provided by all member-countries. The observatory would be controlled by an administration elected by this joint scientific community. We look forward to hearing constructive comments concerning our proposal. (Editors' Note: this proposal was made in the rewriting of Dr. Balega's oral presentation, and therefore there was no specific response from participants at the Colloquium).

TOKOVININ: Perhaps the best way to show the current state of our science is just to speak of the problems of a particular institute. I work at the Sternberg Astronomical Institute, which is part of Moscow University. Although the budget of the institute was increased in 1992, it's actually only one-half of last year's budget when inflation is taken into account. While prices for food are up 10-20 times, our salaries were increased 2 to 4 times and are actually around 1000 rubles per month (or $\$ 10$ U.S. according to the official exchange rate). It was already difficult to live on salary before. Now most of my colleagues have to find additional sources of income in order to survive, especially those with children.

Since 1988 , no instrument developments were funded from the Institute budget. This year the scientific missions and even the postal exchange are severely cut. No currency is allocated for library subscriptions. We are unable to pay the international travel expenses (a "golden curtain" has replaced the iron one).

It is true that, with more than 500 people, our institute has too large a staff. We also have to support our three major observing stations, an old one in Crimea and two just-completed facilities in Central Asia (Alma-Ata in Kazakhstan and Mt. Maidanak in Uzbekistan). The economic difficulties are coupled with the political decisions of the governments of the independent republics to nationalize the property of the former USSR. While the administration in Moscow tries to find ways to keep our observing stations, their local personnel demand greater independence in hope to improve their economic situation on their own.

Astronomy is funded from the state budget as everywhere in the world. The main point of the present government policy is the stabilization of the financial system. Limitation of the budget expenses is given much higher priority than the development or mere protection of science and education. Since overall production has dropped rapidly, there is little hope that the situation of astronomy next year will be any better.

The astronomical community has a long-standing tradition of aiding their eastern-block colleagues. Our presence at this Colloquium is yet another proof of it, and I use this opportunity to thank the SOC for its generous support.

The most desperate need at present is for journal subscriptions. It seems that some help is already promised, and I hope that our library will continue to receive the main astronomical journals in 1992 as a result of the international 
help. Invitations to ex-Soviet astronomers to work abroad for more or less extended periods of time are also important, not only because they help to keep in touch with colleagues and provide access to modern facilities, but also because the savings help to survive when back at home.

It is clear that the international community cannot feed the whole of Soviet astronomy, and the aid must be selective in order to be effective. The groups and individuals that are currently active are easy to identify by publications. The best way to support them is through collaborative research programs and projects. Direct funding of research in Russia is hardly possible due to the bureaucracy, but the support can be made in the form of equipment or in other forms. There are some good examples of such projects that still go on.

ZINNECKER: How do you get to the 6-m telescope? Which is the nearest airport? How do you apply for observing time?

BALEGA: You can apply for observing time twice a year. There is an Allocation Committee of the 6-m Telescope that includes experts in different regions of astrophysical research. One can send an application directly to the Secretary of the Committee, Dr. V. Lipovetski (SAO, Zelenchuk, Stavropol Territory, 357147, Russia). Deadlines for applications are March 1 and September 1. Usually, the first priority is given to those programs which bring new technology to the telescope. To get to the 6-m Telescope, you must first get to Moscow, then to the Mineralniye Vody airport located 100 miles from the Observatory.

ABT: The Astrophysical Journal is sending free subscriptions to the Journal and Supplements to six major centers, including your observatory. Is postal mail a safe method of shipment?

BALEGA: Thank you very much for supporting the 6-m Telescope Observatory! I think that regular mail is still a safe method.

MARSCHALL: Is there any attempt by international or external scientific agencies to fund research directly in the former Soviet Union

TOKOVININ: Not that I know of. However, I know that biologists do have common programs funded from outside the ex-USSR.

ABT: We have heard from Sunyaev that if we try to send money to the CIS, most of it will disappear into taxes.

TOKOVININ: That's right. A better way is to keep money outside the country and to convert it to equipment, travel support, etc.

QUIRRENBACH: One important point seems to be communication. We should perhaps try to identify an appropriate medium (AAS Newsletter?) that could serve as a bulletin board for establishing personal contacts.

TOKOVININ: It's an excellent idea. My colleagues are already very attentive to any information on temporary positions, grants, etc. It is important to know what sources of funding can be potentially available to support the research and, perhaps, to announce calls for cooperation from our side.

McALISTER: Electronic mail to the ex-USSR seems to work most of the time, and computer bulleting boards might provide an easy, convenient, and fast medium toward this goal. 